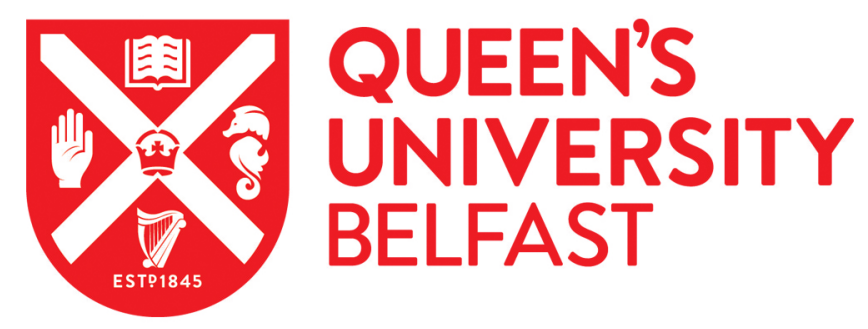

\title{
Interactions between pyrazole derived enantiomers and Chiralcel OJ: Prediction of enantiomer absolute configurations and elution order by molecular dynamics simulations
}

Hu, G-X., Huang, M., Luo, C., Wang, Q., \& Zou, J. (2016). Interactions between pyrazole derived enantiomers and Chiralcel OJ: Prediction of enantiomer absolute configurations and elution order by molecular dynamics simulations. Journal of Molecular Graphics and Modelling, 66, 123-132.

https://doi.org/10.1016/j.jmgm.2016.04.002

\section{Published in:}

Journal of Molecular Graphics and Modelling

\section{Document Version:}

Peer reviewed version

Queen's University Belfast - Research Portal:

Link to publication record in Queen's University Belfast Research Portal

\footnotetext{
Publisher rights

(c) 2016. This manuscript version is made available under a Creative Commons Attribution-NonCommercial-NoDerivs License

(http://creativecommons.org/licenses/by-nc-nd/4.0/), which permits distribution and reproduction for non-commercial purposes, provided the author and source are cited.
}

\section{General rights}

Copyright for the publications made accessible via the Queen's University Belfast Research Portal is retained by the author(s) and / or other copyright owners and it is a condition of accessing these publications that users recognise and abide by the legal requirements associated with these rights.

Take down policy

The Research Portal is Queen's institutional repository that provides access to Queen's research output. Every effort has been made to ensure that content in the Research Portal does not infringe any person's rights, or applicable UK laws. If you discover content in the

Research Portal that you believe breaches copyright or violates any law, please contact openaccess@qub.ac.uk. 
Interactions between pyrazole derived enantiomers and Chiralcel OJ: Prediction

of enantiomer absolute configurations and elution order by molecular dynamics simulations

Guixiang $\mathrm{Hu}^{\mathrm{a}}$, Meilan Huang,*, Chengcai Luo ${ }^{\mathrm{a}}$, Qi Wangc, Jian-Wei Zou ${ }^{\mathrm{a}}$

a School of Biotechnology and Chemical Engineering, Ningbo Institute of Technology, Zhejiang University, Ningbo 315100, China

b School of Chemistry and Chemical Engineering, Queen’s University Belfast, David Keir Building, Stranmillis Road, Belfast BT9 5AG, U.K.

c Minsheng Group Shaoxing Pharmaceutical Co., Ltd, Shaoxing 312071, China

*Corresponding author.

E-mail address: m.huang@qub.ac.uk 
ABSTRACT: The separation of enantiomers and confirmation of their absolute configurations is significant in the development of chiral drugs. The interactions between the enantiomers of chiral pyrazole derivative and polysaccharide-based chiral stationary phase cellulose tris(4-methylbenzoate) (Chiralcel OJ) in seven solvents and under different temperature were studied using molecular dynamics simulations. The results show that solvent effect has remarkable influence on the interactions. Structure analysis discloses that the different interactions between two isomers and chiral stationary phase are dependent on the nature of solvents, which may invert the elution order. The computational method in the present study can be used to predict the elution order and the absolute configurations of enantiomers in HPLC separations and therefore would be valuable in development of chiral drugs.

Keywords: Enantiomer; Solvent Effect; Interaction; Molecular Dynamics (MD); Elution Order 


\section{Introduction}

Chirality is important in drug development because chiral drugs are associated with good curative effect, less toxic side effect and low dosage [1]. The two enantiomers of a drug, such as thalidomide and ibuprofen [2,3], are different in pharmacological activity, acute and chronic toxicity, receptor selectivity, metabolism and excretion rate [4-8]. However the separation of enantiomers and confirmation of their absolute configurations is a long-standing problem [9]. Enantioselective high performance liquid chromatography (HPLC) is one of the most rapid and efficient method for obtaining the two enantiomers of a chiral compound with high optical purity [10]. It is based on the principle that one isomer interacts with the chiral stationary phase (CSP) via three sites, while the other interacts CSP via two-site or less attachment $[11,12]$. Since one enantiomer is more stably attached on the CSP and has the longer eluting time than the other, the two enantiomers are separated. Cellulose tris-(4-methylbenzoate) (Chiralcel OJ), cellulose tris-(3,5-dimethylphenylcarbamate) (Chiralcel OD) and amylose tris-(3,5-dimethylphenalcarbamate) (Chiralpak AD) are three most widely used polysaccharide type CSPs.

Although many chiral compounds can be separated by HPLC effectively [13,14], the absolute configurations of the isomers cannot be confirmed directly [15]. e.g. it was risky to assign the absolute configurations of $\alpha$-arylthiocarboxylic acid analogs on CSP by extrapolation because there is little correlation between elution order and substitution [16]. Therefore the absolute configuration prediction by extrapolation to structural analogs has to be combined with another independent orthogonal method 
[16]. The most common methodologies for absolute configuration assignment nowadays are NMR [17], circular dichroism (CD) spectroscopy [18] and X-ray diffraction, the most preferred direct method to determine absolute configuration [19]. Several complicated methods were used to determine the absolute configurations of the enantiomers such as the combination of Mosher's method with CD spectroscopy [20], the combination of vibrational circular dichroism (VCD), electronic circular dichroism (ECD) measurements and theoretical calculations [21].

Computation techniques have been increasingly used to predict retention and separation factors, to study recognition mechanism and to predict the absolute configurations of eluting peaks combined with HPLC [22-26]. Alcaro et al studied the enantioselective recognition of an asymmetric pyrazole derivative toward Chiralcel OJ stationary phase using docking method [27]. The free energy of complexation was in agreement with the chromatographic values based on the best pose of the docking model. However the recognition of Chiralcel OJ is fulfilled by formation of a transient complex between CSP and an enantiomer which is inserted at different cavities of CSP, i.e., the position of the isomer in the groove changes with the flow of the mobile phase and therefore the interaction between the isomer and the CSP would change accordingly. Therefore, it is necessary to consider different positions where an isomer is located on CSP during elution. In our previous research [28], the interactions between chiral imidazole derivatives and CSP were studied in two solvents and we found that the types of solvents had influence on the interactions between the enantiomers and CSP. Since there is no hydrogen bond donor in imidazole derivatives and CSP [28,29], nonpolar interaction plays a dominate role between chiral imidazole derivatives and CSP. Thus it would be interesting to elucidate how solvents would affect the interactions when a polar group is introduced into the structure. On the other 
hand, separation of chiral drugs by HPLC has been performed under different column temperature [30,31], therefore, it is also important to study if temperature has similar influence on their interactions.

Pyrazole derivatives with a chiral center have potent and selective in vitro activity against monoamine oxidase A and exhibited anti-inflammatory, antitumor, anticancer, antidepressant/antianxiety and antinociceptive effect [32-34]. It is well-known that mobile phase has significant influence on the separation of enantiomers by HPLC $[35,36]$. At room temperature $\left(25^{\circ}\right)$, 1-acetyl-3-(4-hydroxyphenyl)-5-(2-chlorophenyl)-4,5-dihydro-(1H)-pyrazole (compound $\mathbf{1}$ in Fig. 1) was separated using four types of mobile phases on chiralcel OJ (Fig. 1) [37]. In contrast to imidazole derivatives, there is a polar $\mathrm{OH}$ group in compound $\mathbf{1}$ and the (R)-enantiomer was eluted first followed by the (S)-enantiomer in all mobile phases [37]. In this paper, systematic study was performed to investigate the influence of solvents and temperature on the separation of the two enantiomers of the pyrazole derived compound. Each isomer was positioned at three different positions on a CSP column to reflect the positions during elution.

\section{Computational methods}

\subsection{Parameter setting}

Polymer Consistent Force Field (PCFF) derived from CFF91 was employed in all computations [38]. It was specifically developed for polymers such as polycarbonates and polysaccharides, as well as organic materials [39-43]. The total potential energy is calculated by Equation (1).

$$
\begin{aligned}
E= & E_{b}+E_{\theta}+E_{\phi}+E_{\chi}+E_{b b^{\prime}}+E_{\theta \theta^{\prime}}+E_{b \theta}+E_{b \phi}+E_{b^{\prime} \phi}+E_{\theta \phi}+E_{\phi \theta \theta^{\prime}} \\
& +\sum_{i>j} \frac{q_{i} q_{j}}{\varepsilon r_{i j}^{P}}+\sum_{i>j}\left[\frac{A_{i j}}{r_{i j}^{9}}-\frac{B_{i j}}{r_{i j}^{6}}\right]
\end{aligned}
$$


The first eleven items are chemical bond energies and the last two items are non-bonded interaction energies, namely electrostatic energy and van der Waals energy. The electrostatic energy is computed by the cell multipole method [44], which is rigorous and efficient in handling non-bonded interactions in both non-periodic and periodic systems. The van der Waals energy is based on Lennard-Jones potential to calculate long-range non-bonded interactions [45]. All calculations were performed using the Discover module in the Material Studio software (Accelrys, USA). The molecular dynamics (MD) simulation and structure optimization were performed using NVT ensemble and Andersen thermostat [46].

2.2. Preparation of complex conformations and calculation of bindinginteraction energies

The structures of the enantiomers and the monomer of Chiralcel OJ were built in Material Studio and optimized using conjugate gradient algorithm [47] and Polak-Ribiere method [48]. Because cellulose is a highly ordered helical natural polymer and a big helix are formed by 12 monomers [28], a model composed of 12 monomers was built based on the optimized monomer of Chiralcel OJ with the terminal replaced by methyl (Fig. 2a). The coordinates of the 12-mer Chiralcel OJ can be downloaded from supplementary information. In MD simulations, all individual enantiomer and CSP were subjected to 100ps equilibration at 500K followed by another 100ps production at 298K. Conformations were saved every 10ps. Thus 10 frames were generated from the production and each of them was subsequently minimized. The convergence threshold was set to $0.1 \mathrm{kcal} /(\mathrm{mol} \cdot \AA)$ and the dielectric constant (DC) set to 1.00. The lowest energy conformations of the enantiomers and CSP were used to build the enantiomer-CSP complex model.

Each isomer was placed into the groove of the side chain of Chiralcel OJ to build 
the initial complex model. Because the position of the isomer in the groove and the interaction between the isomer and the CSP changes with the flow of the mobile phase, each isomer was positioned at three different initial positions (Fig. 2b). One was located in the middle groove of CSP and the other two were slightly towards each end of the CSP.

MD was performed for each initial complex. First, 100ps run was carried out at $500 \mathrm{~K}$. Then the structures were equilibrated at 298K (the column temperature) for 200ps followed by 100ps production. Conformations were saved every 10ps. Thus 10 conformations were retrieved at each of the three interaction positions, resulting in 30 conformations. These conformations were then subject to minimization with convergence threshold set to $0.1 \mathrm{kcal} /(\mathrm{mol} \cdot \AA)$.

The bindinginteraction energy ( $\left.E_{\text {binding }} E_{\text {interact }}\right)$ between an isomer and the CSP was calculated based on the energies of the complex, the CSP and the isomer ( $E_{\text {complex, }}$ $E_{\text {CSP }}$ and $E_{\text {isomer }}$ (Equation (2)) [49,50].

$$
E_{\text {interact }}=E_{\text {complex }}-\left(E_{C S P}+E_{\text {isomer }}\right)
$$

\subsection{Solvent effect}

Solvent effect mainly includes short-range and long-range effect. The former refers to the covalent or hydrogen bond interactions between solvent and solute. The latter refers to the electrostatic interactions between the dipoles of solvent and solute. In the present study the DC values were set to highlight the long-range effect of various solvents on the interactions between the isomers and CSP. Compound $\mathbf{1}$ was separated with four types of mobile phases, n-hexane/ethanol (70/30), n-hexane/2-propanol (60/40), ethanol and 2-propanol. The DC values are 9.06, 8.58, 
25.80 and 18.62 respectively. In addition, three reference solvent conditions, vacuum, n-hexane and water with DC values of $1.00,1.89,81.00$, respectively, were also considered in order to explore the solvent effect systematically.

The interaction energies between the isomers and CSP are affected by initial conformations and solvents. In order to examine the influence of initial conformations on the bindinginteraction energies, compound 1 was placed at three different positions in the CSP groove in four types of mobile phases, n-hexane/ethanol (70/30), n-hexane/2-propanol (60/40), ethanol and 2-propanol. Furthermore, in order to examine the net solvent effect, the three initial conformations in the three reference solvents (vacuum, n-hexane and water) were set to the same as that in 2-propanol.

\subsection{Temperature effect}

The column temperature also has an important effect on HPLC separation [30, 31]. Therefore, in addition to the experimental temperature of $298 \mathrm{~K}$, calculations were also performed at high temperature (500K) and low temperature (100K) under the mobile phase 2-propanol (DC = 18.62) following the same protocol as that applied for the room temperature. In order to evaluate the net temperature effect, the compound 1 at these temperature conditions was placed at three positions same as those set for 298K.

MD simulation was performed for each initial complex. First 100ps run was carried out at $500 \mathrm{~K}$ to overcome the molecular energy barrier-firstly. Then the structures were equilibrated at $500 \mathrm{~K}$ or $100 \mathrm{~K}$ for $200 \mathrm{ps}$ followed by $100 \mathrm{ps}$ production at the samerespective temperature. The conformations of complex were saved every $10 \mathrm{ps}$, so 10 conformations were retrieved for every interaction position, which led to 30 conformations for three positions. These conformations were then subjected to minimization with convergence threshold set to $0.1 \mathrm{kcal} /(\mathrm{mol} \cdot \AA)$.

Commented [m1]: 对于两个不同温度,

500K： 系统先升温到 $500 \mathrm{~K}$ 以克服能垒, 然后在 $500 \mathrm{~K}$ 平衡 200ps?

100K：这个模拟也是先升温到 $500 \mathrm{~K}$, 然后再降到 $100 \mathrm{~K}$ 平衡 200ps

是的, 就相当于前面 $298 \mathrm{~K}$ 那部分, 在 $298 \mathrm{~K}$ 的温度改成 $500 \mathrm{~K}$, 或者 $100 \mathrm{~K}$, 其实就是平衡温度和 production 温度分别设置为 298 , 500,100 . 这部分和前面的重复了, 但审稿人没弄明白, 只能在这 里重复叙述一下了。 


\section{Results and discussion}

\section{1. $M D$ simulations}

Chiralcel OJ belongs to polysaccharides, so PCFF, a force field specially developed for polymers was used in all computations. The first 100ps run was performed at $500 \mathrm{~K}$ to allow the complexes to overcome energy barriers such that the total potential energy hypersurface was fully explored. Fig. 3 shows the relationship between the energy and the simulation time for the (R)- isomer in the mobile phase 2-propanol. At 500K, the system reached the equilibration after 25ps. At 298K, the energy of the system fell quickly and leveled off. Rest of the simulation results were similar to those described here.

\subsection{Energy analysis}

\subsubsection{Solvent effect}

It was reported that the (R)- enantiomer of the compound $\mathbf{1}$ was eluted first followed by the (S)- isomer when they were separated using four types of mobile phases, n-hexane/ethanol (70/30), n-hexane/2-propanol (60/40), ethanol and 2-propanol [37]. Fig. 4 shows the calculated interaction energies in seven solvents. The interaction energy values are listed in Table S1. It is demonstrated that the mean interaction energy of the 30 conformations of the (S)- enantiomer is lower than that of the (R)- enantiomer in polar solvents, no matter it is a mixture solvent such as n-hexane/ethanol (70/30) and n-hexane/2-propanol (60/40) or a pure solvent such as ethanol, 2-propanol and water. That is, the interaction between the (S)- isomer and the CSP is stronger than the (R)- isomer such that the (R)- isomer elutes first followed by the (S)- isomer when the enantiomers are separated by HPLC. This is coincident with 
the experimental results reported in previous literature [37]. In contrast, the interaction between the (R)- isomer and the CSP is stronger than the (S)- isomer in vacuum or non-polar solvent n-hexane, indicating that the (S)- isomer elutes first followed by the (R)- isomer. It should be noted that the initial structures of the complex in vacuum and n-hexane are the same as those in 2-propanol and water. Thus the different features of the solvents decide the preference of the isomers on the CSP, and therefore have a significant influence on the separation results. So the DC value in MD simulations should be set in accordance with the HPLC mobile phase so that results can be used to accurately predict the absolute configurations of the enantiomers under the experimental separation conditions.

Table 1 shows the decomposition of the interaction energy of the mean energy conformation (the conformation whose energy is closest to the mean energy). The bindinginteraction energies and long-range van der Waals interactions are calculated by Equations (3) and (4).

$$
\begin{aligned}
& E_{\text {interact }}=E_{v d w}+E_{\text {ele }} \\
& E_{v d w}=E_{r e p}+E_{d i s p}
\end{aligned}
$$

It can be seen that the contribution of the van der Waals energy is much greater than the electrostatic energy while the order of the van der Waals energies of (R)- and (S)- configurations is coincident with that of their bindinginteraction energies. With an increase in the polarity of the mobile phases, the contribution of the electrostatic energy decreases for both enantiomers. This is because the polar electrostatic interaction between the isomers and CSP is more obvious in nonpolar environment, whereas the polar interaction is weakened in polar environment. Van der Waals interaction is composed of repulsive energy and dispersive energy. When two 
molecules are close to each other, the steric repulsive energy leads to the instability and strong dispersive energy results in the stability of the complex. The complex tends to an equilibrium state under the two opposite interaction energies. Although the repulsive energy makes the configurations unstable in all solvents, the stronger dispersive energy bring the complexes into stable states (Table 1).

It is well-known that DC has influence on electrostatic interaction but shows no effect on van der Waals interaction [29]. The complex conformations of the (R)- and (S)- isomer and CSP from the same initial conformation (middle) in 2-propanol and vacuum are shown in Fig.5. The conformations were obtained from a 100-ps MD simulation at high temperature (500 K) and 200-ps equilibration. Although the isomer is located in the groove of CSP in 2-propanol (Fig. 5a), there is no strong interaction between the groups of the (R)- isomer and CSP. Whereas the interactions between the isomer and CSP are stronger in vacuum than in 2-propanol with a hydrogen bond formed between the hydroxyl group of the isomer and the carbonyl group of CSP (Fig. 5c). Similar results were obtained between the (S)- isomer and CSP (Fig. 5b and Fig. 5d). So starting from the same initial conformation, the complex can evolve into different conformations in different solvents. Therefore, solvent condition not only decides the elution order of the enantiomers, but also has significant impact on the dynamic evolution of the complexes and their stability.

\subsubsection{Temperature effect}

Fig. 6 shows the calculated interaction energies under three temperature conditions. The interaction energies are listed in Table S2. It can be seen that the average interaction energy of the (R)- isomer is weaker than that of the (S)- isomer at $100 \mathrm{~K}$ and $298 \mathrm{~K}$, which suggests that the (R)- isomer elutes faster than the (S)- isomer. It should be noted that the interaction energies of the two enantiomers are very close 
to each other (the difference is less than $1 \mathrm{kcal} / \mathrm{mol}$ ) at a high temperature of $500 \mathrm{~K}$, although the initial conformations are set to the same as those at $100 \mathrm{~K}$ and $298 \mathrm{~K}$. This implies the enantiomers cannot be separated effectively at high temperature. Starting from the same initial complex conformation, the two enantiomers exhibit different separation results under different temperature conditions.

Temperature influences the thermodynamic retention and separation of the enantiomers and their kinetic diffusion [51]. In chromatographic enantioseparation, the relationship between retention factor, separation factor and temperature can be described by the Van’t Hoff equation (Equations (5) and (6)) [31,51].

$$
\begin{array}{r}
\ln k=-\frac{\Delta H^{0}}{R T}+\frac{\Delta S^{0}}{R}+\ln \Phi \\
\ln a=-\frac{\Delta \Delta H^{0}}{R T}+\frac{\Delta \Delta S^{0}}{R}
\end{array}
$$

In the equations, $k$ is retention factor and $a$ is separation factor. $\Delta H^{0}$ and $\Delta S^{0}$ represent the differences in the enthalpy and entropy when an isomer transfers from mobile phase to CSP. $R$ is universal gas constant and $\Phi$ is phase ratio. $\Delta \Delta H^{0}$ and $\Delta \Delta S^{0}$ represent the differences of $\Delta H^{0}$ and $\Delta S^{0}$ for a given pair of enantiomers, respectively. Positive $\Delta \Delta H^{0}$ and $\Delta \Delta S^{0}$ value suggests that the enantioseparation is entropy-driven and the separation factor increases with an increase in temperature. Negative $\Delta \Delta H^{0}$ and $\Delta \Delta S^{0}$ values mean that enantioseparation is enthalpy-driven and the separation factor decreases with an increase in temperature [52]. A turning temperature exists and for the enthalpy-driven enantioseparation, a temperature lower than the turning temperature should be chosen. Since the separation of the compound $\mathbf{1}$ on chiralcel OJ is enthalpy-driven [53], high temperature is disadvantageous to the separation. In addition, the difference of bindinginteraction energies between the (R)and (S)- isomer at $100 \mathrm{~K}$ is smaller than that at $298 \mathrm{~K}$. In the previous literature, Zhou R. et. al reported poor enantioseparation of Metoprolol on BCDSP at low temperature 
[51]. The negligible difference in the bindinginteraction energies of the two enantiomers at low temperature may be caused by slow diffusion which further leads to long retention time and weak separation.

Although temperature effect is not as significant as solvent effect, it cannot be ignored in the simulation. Therefore the simulated temperature should be set based on the actual column temperature in HPLC experiment.

The interaction energies of the mean-energy conformations are listed in Table 2. Similar to the solvent effect, under different temperature conditions, the contribution of the van der Waals energy is much greater than that of the electrostatic energy and the order of the van der Waals energies of the (R)- and (S)- configurations is coincident with their bindinginteraction energies. Meanwhile, the difference in the interaction energies between the two isomers at $100 \mathrm{~K}$ and $298 \mathrm{~K}$ is mainly attributed to the dispersive energy.

\subsection{Structure analysis}

The plot of retention factor vs. solvent ratio disclosed a U-shaped retention curve, indicating that different interactions are dominant in different mixture of polar organic solvents [54]. It was suggested that the main sites of Chiralcel OJ involved in the chiral separation are probably the polar carbonyl groups and the aromatic fragments of the ester functional group [53]. The chiral compound $\mathbf{1}$ contains an acetyl substituted pyrazole and a phenol ring while CSP contains a 4-methylbenzoate side chain (Fig. 1). The carbonyl groups of the CSP are located inside the polymer chain and can act as hydrogen bond acceptors and establish dipole-dipole interactions with the enantiomers. The aromatic fragments are located outside the polymer chain and 
can form $\pi-\pi$ or $\mathrm{CH}_{3}-\pi$ interactions with the enantiomers.

From the computational results and the structures of the chiral compound $\mathbf{1}$ and Chiralcel OJ, we suggest that the interactions between the two enantiomers and CSP are mainly dominated by nonpolar steric interactions such as $\pi$ - $\pi$ stack or hydrophobic interactions formed between the alkyl groups of the CSP and the aromatic ring of the enantiomers in the polar solvents. The distinction of the nonpolar interactions between the two enantiomers and CSP becomes more remarkable against strong polar solvent surroundings. So the enantiomers can be separated effectively in the polar solvents. When the polarity of the solvents is weakened, the nonpolarity of the surroundings attenuates the marginal difference of the nonpolar interactions between the two isomers and CSP. The decreased separation effect even makes the difference disappear, thus the enantiomers cannot be separated effectively.

This actually is in accordance with previous experimental observations on the enantioseparation of various chiral compounds. For example, a chiral 3,5-diarylpyrazole derivative (compound 2 in Fig.1) was separated effectively on Chiralcel OJ in polar solvents [55], since the nonpolar interactions between the enantiomers and CSP dominate and polar solvents are favourable to the separation of the enantiomers. In ethanol eluent, the hydrophobic group in compound $\mathbf{2}$ makes the separation factor increase on Chiralcel OJ and the (R)-configuration elutes first [53]. Similar result was also observed on other types of CSPs. For example, Terazosin (compound 3 in Fig. 1) was separated effectively in polar solvents on Chiralpak IC (cellulose tris(3,5-dichlorophenylcarbamate)) [56].

In addition, we suggest that the nonpolar solvents are beneficial to the separation of enantiomers when polar interactions dominate between the enantiomers and CSP. This again is supported by previous experimental results. For instance, baseline 
separation of azelnidipine (compound $\mathbf{4}$ in Fig.1) was achieved in mobile phase n-hexane/2-propanol (90/10) (DC =3.56) on Chiralpak AD (amylose tris(3,5-dimethylphenylcarbamate)) [57]. The separation factor of chiral oxaliplatin (compound 5 in Fig.1) decreased with the enhancement of the polarity of the mobile phase which even reached 1 on Chiralpak IC [58]. Two neonicotinoid pesticides compound 6 and 7 (Fig. 1) were separated on Chiralcel OD. The separation factor of compound $\mathbf{6}$ decreased whereas compound $\mathbf{7}$ increased, when the polarity of mobile phase was lost [59]. This indicated that the polar interactions between compound 7 and CSP were stronger than compound $\mathbf{6}$, because there are more polar groups in compound 7 than 6 .

On the other hand, strong nonpolarity of the surroundings may manifest the few polar hydrogen bonds interactions between the enantiomers and CSP and may result in the inversion of elution order. In the previous study [28], a chiral imidazole derivative was separated in n-hexane/ethanol (50/50), while the distinction of interaction between the two enantiomers disappeared in n-hexane/ethanol (98.5/1.5). In the present study, the elution order was inversed in nonpolar solvent n-hexane or in vacuum when the DC value was further decreased to 1.89 and below. So it can be deduced that a threshold DC value exists under which the polar and nonpolar interactions between enantiomers and CSP would counteract each other, as a result the distinction between enantiomers disappears during HPLC separation. In the polar solvents with DC values higher than the threshold DC, the nonpolar interaction dominates so that the isomers can be separated effectively; in the nonpolar solvents with DC values lower than the threshold DC, the polar interaction dominates and the isomers also can be separated, although the elution order maybe inverted.

For example, an imidazole derivative (compound 8 in Fig. 1) was separated and 
the (+)-isomer eluted first in ethanol/diethylamine (100/0.1) solvent, while the (-)-isomer eluted first in 2-propanol/diethylamine (100/0.1) solvent on Chiralcel OJ [29]. Similar observations were also reported on other CSPs. Compound 1 (Fig. 1) was separated on Chiralpak AD and the (R)- isomer eluted first in n-hexane/2-propanal (60/40) mobile phase, while the (S)- isomer eluted first in n-hexane/ethanol (70/30) mobile phase [37]. The (+)-isomer of an analogue (compound $\mathbf{9}$ in Fig. 1) of compound 1 eluted first in 2-propanol solvent, while its (-)-isomer eluted first in ethanol solvent on Chiralcel OD[37]. The elution order of racemic Omeprazole, Pantoprazole, Lansoprazole and Rabeprazole was inverted in different mobile phases on Chiralpak IA (amylase tris(3,5-dimethylphenylcarbamate)) [60]. A spiral compound, 2,2'-di-(n-butyl)-1,1'-bibenzimidazole (Compound 10 in Fig. 1) was separated and the P- isomer eluted first in n-hexane/2-propanol (100/10) solvent, while the M- isomer eluted first in n-hexane/ethanol (100/10) solvent on Chiralpak AD [61]. Similar observation was reported for the separation of chiral polyhalogenated 4,4'-bipyridines (Compound 11 in Fig. 1) in different solvents on polysaccharide carbamate-based CSP [62].

The mean energy conformations of the complexes in 2-propanol (DC =18.62) are shown in Fig. 7. We found although the (R)- isomer is embedded in the groove, 5-(2-chlorophenyl) is bare and its benzene ring is almost vertical to that in the CSP (Fig. 7a). The (S)- isomer is also embedded in the groove and surrounded by the side chains of the CSP and $\pi$ - $\pi$ stack is formed between the 5-phenyl of the isomer and the benzene ring of the CSP (Fig. 7b). So the interaction energy between the (R)- isomer and CSP is weaker than that of the (S)- isomer. Fig. 7c and Fig. 7d show the conformation of the complexes with energy closest to the mean energy in vacuum (DC =1.00). In non-polar solvent, it is obvious that the $(\mathrm{R})$ - isomer is embedded in the 
groove binding more tightly than the (S)- one. The mean energy conformations of the complexes in other solvents are shown in Fig. S1. All of the complex structures were retrieved from the MD simulations in different solvents at 298K.

Complex structures were also retrieved from the simulations under different temperature conditions in 2-propanol ( $\mathrm{DC}=18.62$ ). The mean energy conformations of the complexes at 500K are shown in Fig. 7e and Fig. 7f. In the complex of the (R)isomer and the CSP, the benzene ring of 3-(4-hydroxyphenyl) is located between the methyl and the aromatic ring of the CSP forming a $\mathrm{CH}_{3}-\pi-\pi$ interaction. The 5-(2chlorophenyl) group shows no obvious interaction with CSP. In the complex of the (S)- isomer and the CSP, a $\mathrm{CH}_{3}-\pi$ interaction is formed between the methyl of 1-acetyl and the benzene ring of the CSP along with a $\pi-\pi$ interaction formed between the benzene rings of 3-(4-hydroxyphenyl) and the CSP. The similar interaction between the two isomers and CSP indicates there is little difference in their bindinginteraction energies. Therefore the enantiomers cannot be separated effectively at high temperature. The mean energy conformations of the complexes in 2-propanol $(\mathrm{DC}=18.62)$ at $100 \mathrm{~K}$ are shown in Fig. S2.

Although same initial conformations were employed in various mobile phases (with DC values of $1.00,1.89,18.62$ and 81.00 ) and under extreme temperature conditions (500K and $100 \mathrm{~K})$, it is worth noting that a hydrogen bond is only observed in the conformations of the (R)- and (S)- enantiomers in vacuum (DC =1.00) and the (S)- enantiomer in n-hexane (DC =1.89) among all complexes (Fig. 7, Fig. S1). This validates our suggestion that the dominating interactions between the two enantiomers and CSP may change from the nonpolarity to the polarity when the polarity of 
solvents is decreased.

\section{Conclusions}

The separation of chiral drug enantiomers and confirmation of their absolute configurations are significant in the development of drugs. The pyrazole derivative chiral compound $\mathbf{1}$ is a potent anti-inflammatory, antitumor, antidepressant and antinociceptive drug. Systematic study was performed on the interactions between the enantiomers of the compound and the CSP (Chiralcel OJ) in seven solvents and three temperature conditions using molecular dynamics simulations. The results disclose that the solvent effect has remarkable influence on the interaction. In polar solvents, the complex of the (S)- isomer and the CSP is more stable than that of the (R)- isomer, which makes (R)- isomer elute first. This is accordant with previous experimental results. In the hypothetical nonpolar solvents ( $\mathrm{DC}=1.00$ or 1.89 ), the elution order is opposite to that observed in the polar solvents. The interaction energy between the (R)- isomer and the CSP is stronger than that of the (S)- isomer, which makes the (S)isomer elute first. Same initial structures of the complex were used in vacuum, n-hexane, 2-propanol and water, respectively. Interestingly, the elution order of the enantiomers was inverted in polar solvents in contrast to the results observed under nonpolar condition. Structure analysis demonstrates that the distinction of nonpolar interactions between the two isomers and CSP are more remarkable in polar solvents. While in nonpolar solvents, the distinction of polar interactions between the two isomers and CSP are more remarkable. The different interactions in various polar solvents may make the elution order invert. Temperature effect on the separation result was also investigated by computational study. Energy analysis shows that the intermolecular van der Waals energy, especially the dispersive energy determines the 
separation efficiency and the elution order of the enantiomers on the stationary phase. This computational method in the present study opens can be used to predict the elution order and the absolute configurations of the peaks in HPLC enantioseparations.

\section{Acknowledgements}

The authors are grateful to INVEST NI (INI RD0314092), the National Natural Science Foundation of China (21002088, 21272211), Ningbo Natural Science Foundation (2014A610118) and Program of Science and Technology of Ningbo, China (2013D1003) for financial supports.

Supplementary Information Available: [The coordinates and three-dimension structure of the 12-mer Chiralcel OJ, the conformations of the (R)-/(S)- isomers with energy closest to the mean energy at $298 \mathrm{~K}$ and 100K, respectively.]

\section{References}

1. L. A. Nguyen, H. He, C. Pham-Huy, Chiral Drugs: An Overview, Int. J. Biomed. Sci. 2 (2006) 85-100.

2. S. J. Matthews, C. McCoy, Thalidomide: A Review of Approved and Investigational Uses, Clin. Ther. 25 (2003) 342-395.

3. K. Doki, T. Hayakawa, W. Lin, H. Yanagimoto, G. Ding, N. Inotsume, Effects of Absorption Rate on the Pre-systemic Chiral Inversion of Ibuprofen in Rabbits, J. Pharm. Pharmacol. 55 (2003) 1091-1097.

4. M. R. Linder, A. R. Heckeroth, M. Najdrowski, A. Daugschies, D. Schollmeyer, C. Miculka,(2R, 3S)-(+)-and (2S, 3R)-(-)-Halofuginone Lactate: Synthesis, Absolute Configuration, and Activity Against Cryptosporidium Parvum, Bioorg. Med. Chem. Lett. 17 (2007) 4140-4143.

5. M. M. Lordelo, M. C. Calhoun, N. M. Dale, M. K. Dowd, A. J. Davis, Relative Toxicity of Gossypol Enantiomers in Laying and Broiler Breeder Hens, Poult. Sci. 86 (2007) 582-590.

6. G. Pochetti, C. Godio, N. Mitro, D. Caruso, A. Galmozzi, S. Scurati, F. Loiodice, 
G. Fracchiolla, P. Tortorella, A. Laghezza, A. Lavecchia, E. Novellino, F. Mazza, M. Crestani, Insights into the Mechanism of Partial Agonism, J. Biol. Chem. 282 (2007) 17314-17324.

7. H. Lu, Stereoselectivity in Drug Metabolism, Expert Opin. on Drug Metab.Toxi. 3 (2007) 149-158.

8. A. R. M. de Oliveira, P. S. Bonato,Stereoselective Determination of Hydroxychloro-quine and Its Major Metabolites in Human Urine by Solid-phase Microextraction and HPLC, J. Sep. Sci. 30 (2007) 2351-2359.

9. N. M. Maier, P. Franco, W. Lindner, Separation of Enantiomers: Needs, Challenges, Perspectives, J. Chromatogr. A 906 (2001) 3-33.

10. Y. Okamoto, T. Ikai, Chiral HPLC for Efficient Resolution of Enantiomers, Chem. Soc. Rev. 37 (2008) 2593-2608.

11. R. Bentley, Diastereoisomerism, Contact Points, and Chiral Selectivity: A Four-site Saga, Arch. Biochem. Biophys. 414 (2003) 1-12.

12. M. Lämmerhofer, Chiral Recognition by Enantioselective Liquid Chromatography: Mechanisms and Modern Chiral Stationary Phases, J. Chromatogr. A 1217 (2010) 814-856.

13. Y. Zhang, S. Yao, H. Zeng, H. Song, Chiral Separation of Pharmaceuticals by High Performance Liquid Chromatography, Curr. Pharm. Anal. 6 (2010) 114-130.

14. M. X. Du, X. H. Qian, L. P. Wang, X. Shao, W. Z. Li, Enantioseparation of Chiral Alcohols on A Cellulose Derivative Immobilized onto Spherical Mesoporous SBA-15 by High Performance Liquid Chromatography, Chem. J. Chinese Universities 33 (2012) 902-907.

15. Z. Benfodda, D. Benimelis, M. Jean, J. V. Naubron, V. Rolland, P. Meffre, Synthesis, Resolution, and Determination of Absolute Configuration of Protected $\alpha$-Ethynylphenylalanine Enantiomers, Amino Acids 47 (2015) 899-907.

16. M. Lämmerhofer, R. Pell, M. Mahut, M. Richter, S. Schiesel, H. Zettl, M. Dittrich, M. Schubert-Zsilavecz, W. Lindner, Enantiomer separation and indirect chromatographic absolute configuration prediction of chiral pirinixic acid derivatives: Limitations of polysaccharide-type chiral stationary phases in comparison to chiral anion-exchangers. Journal of Chromatography. A 1217 (2010) 1033-1040. 
17. T. Kurtan, N. Nesnas, F. E. Koehn, Y.-Q. Li, K. Nakanishi, N. Berova, Chiral Recognition by CD-Sensitive Dimeric Zinc Porphyrin Host. 2. Structural Studies of Host-Guest Complexes with Chiral Alcohol and Monoamine Conjugates. J. Am. Chem. Soc. 123 (2001) 5974-5982.

18. N. Harada, M. Watanabe, S. Kuwahara, A. Sugio, Y. Kasai, A. Ichikawa, 2-Methoxy-2-(1-naphthyl)propionic acid, a powerful chiral auxiliary for enantioresolution of alcohols and determination of their absolute configurations by the 1H NMR anisotropy method. Tetrahedron : Asymmetry 11 (2000) 1249-1253.

19. H. D. Flack, G. Bernardinelli, The Use of X-ray Crystallography to Determine Absolute Configuration, Chirality 20 (2008) 681-690.

20. R. Cirilli, R. Ferretti, G. L. Regina, G. Morrelli, M. Pierini, F. Piscitelli, R. Silvestri, Enantioselective HPLC Combined with Spectroscopic Methods: A Valid Strategy to Determine the Absolute Configuration of Potential-secretaseInhibitors, Talanta 82 (2010) 1306-1312.

21. N. Vanthuyne, C. Roussel, J. Naubron, N. Jagerovic, P. M. Lazaro, I. Alkorta, J. Elguero, Determination of the Absolute Configuration of 1,3,5-Triphenyl-4,5-Dihydropyrazole Enantiomers by A Combination of VCD, ECD Measurements, and Theoretical Calculations, Tetrahedron: Asymmetry 22 (2011) 1120-1124.

22. M. H. Fatemi, Predictions of Retention Factors for Some Organic Nucleuphiles in Complexation Gas Chromatography, J. Chromatogr. Sci. 49 (2011) 476-481.

23. G. X. Hu, C. C. Luo, S. F. Pan, Y. J. Jiang, J. W. Zou, Predicting Retention and Separation Factors of Chiral Diarylmethane Derivates by QSPR Models, Acta Phys. -Chim. Sin. 31 (2015) 73-82.

24. M. Szaleniec, A. Dudzik, M. Pawul, B. Kozik, Quantitative Structure Enantioselective Retention Relationship for High-performance Liquid Chromatography Chiral Separation of 1-Phenylethanol Derivatives, J. Chromatogr. A 1216 (2009) 6224-6235.

25. A. Del Rio, Exploring Enantioselective Molecular Recognition Mechanisms with Chemoinformatic Techniques, J. Sep. Sci. 32 (2009) 1566-1584.

26. M. Okamoto, Reversal of Elution Order During the Chiral Separation in High 
Performance Liquid Chromatography, J. Pharm. Biomed. Anal. 27 (2002)

401-407.

27. S. Alcaro, A. Bolasco, R. Cirilli, R. Ferretti, R. Fioravanti, F. Ortuso, Computer-Aided Molecular Design of Asymmetric Pyrazole Derivatives with Exceptional Enantioselective Recognition toward the Chiralcel OJ-H Sationary Phase, J. Chem. Inf. Model. 52 (2012) 649-654.

28. G. X. Hu, C. C. Luo, K. L. Yin, J. W. Zou, Q. S. Yu, Influence of Solvent Effect on Interaction between Chiral Imidazole Derivates Enantiomer and Stationary Phase, Chem. J. Chinese Universities 34 (2013) 1497-1504.

29. R. Cirilli, R. Ferretti, B. Gallinella, F. La Torre, G. La Regina, R. Silvestri, Comparative Study between the Polysaccharide-based Chiralcel OJ and Chiralcel OD CSPs in Chromatographic Enantioseparation of Imidazole Analogues of Fluoxetine and Miconazole, J. Sep. Sci. 28 (2005) 627-634.

30. T. Aral, H. Aral, B. Ziyadanogullari, R. Ziyadanogullari, Synthesis of A Mixed-model Stationary Phase Derived from Glutamine for HPLC Separation of Structurally Different Biologically Active Compounds: HILIC and Reversed-phase Applications, Talanta 131 (2015) 64-73.

31. R. N. Rao, K. N. Kumar, Thermodynamic Evaluation of Immobilized Cellulose Tris(3,5-dichlorophenylcarbamate) as A Stationary Phase for Liquid Chromatographic Separation of Darunavir Enantiomers, J. Chromatogr. Sci. 53 (2015) 295-301.

32. T. Zhang, D. Nguyen, P. Franco, Reversed-phase Screening Strategies for Liquid Chromatography on Polysaccharide-derived Chiral Stationary Phases, J. Chromatogr. A 1217 (2010) 1048-1055.

33. R. Cirilli, V. Orlando, R. Ferretti, L. Turchetto, R. Silvestri, G. D. Martino, F. L. Torre, Direct HPLC Enantioseparation of Chiral Aptazepine Derivatives on Coated and Immobilized Polysaccharide-based Chiral Stationary Phases, Chirality 18 (2006) 621-632.

34. M. Celano, S. Schenone, D. Cosco, M. Navarra, E. Puxeddu, L. Racanicchi, C. Brullo, E. Varano, S. Alcaro, E. Ferretti, G. Botta, S. Filetti, M. Fresta, M. Botta, D. Russo, Cytotoxic Effects of A Novel Pyrazolopyrimidine Derivative Entrapped in Liposomes in Anaplastic Thyroid Cancer Cells in vitro and in Xenograft Tumors in vivo, Endocr.-Relat. Cancer 15 (2008) 499-510.

35. F. Chimenti, A, Bolasco, F. Manna, D. Secci, P. Chimenti, O. Befani, P. Turini, V. 
Giovannini, B. Mondovi, R. Cirilli, F. La Torre, Synthesis and Selective Inhibitory Activity of 1-Acetyl-3,5-diphenyl-4,5-dihydro-(1H)-pyrazole Derivatives Against Monoamine Oxidase, J. Med. Chem. 47 (2004) 2071-2074.

36. Z. S. Sahin, U. Salgin-Goksen, N. Gokhan-Kelekci, S. Isik, Synthesis, Crystal Structures and DFT Studies of 1-[2-(5-Methyl-2-benzoxazolinone-3-yl)acetyl] -3-phenyl-5-(3,4-dimethoxyphenyl)-4,5-dihydro-1H-pyrazole and1-[2-(5-chloro2-benzoxazolinone-3-yl)acetyl]-3-phenyl-5-(4-methoxyphenyl)-4,5-dihydro-1Hpyrazole, J. Mol. Struct. 1006 (2011) 147-158.

37. R. Cirilli, R. Ferretti, B. Gallinella, L. Turchetto, A. Bolasco, D. Secci, P. Chimenti, M. Pierini, V. Fares, O. Befani, F. La Torre, Enantiomers of C5-chiral 1-Acetyl-3,5-diphenyl-4,5-dihydro-(1H)-pyrazoleDerivatives: Analytical and Semipreparative HPLC Separation, Chiroptical Properties, Absolute Configuration, and Inhibitory Activity Against Monoamine Oxidase, Chirality 16 (2004) 625-636.

38. H. Sun, S. J. Mumby, J. R. Maple, A. T. Hagler, An Ab Initio CFF93 All-atom Force Field for Polycarbonates, J. Am. Chem. Soc. 116 (1994) 2978-2987.

39. R. Rahman, J. T. Foster, A. Haque, Molecular Dynamics Simulation and Characterization of Graphene-cellulose Nanocomposites, J. Phys. Chem. A 117 (2013) 5344-5353.

40. M. Kavoos, Z. Esmaeil,Investigation of Adsorption of Polymers on Metallic Nanowires: A Molecular Dynamics Strudy, Appl. Surf. Sci. 261 (2012) 242-246.

41. H. Heinz, T. Lin, R.K. Mishra, F.S. Emami,Thermodynamically Consistent Force Fields for the Assembly of Inorganic, Organic, and Biogical Nanostructures: the INTERFACE Force Field, Langmuir 29 (2013) 1754-1765.

42. R. Mishra, L. Femandez-Carrasco, R. J. Flatt, H. Heinz, A Force Field for Tricalcium Aluminate to Characterize Surface Properties, Initial Hydration, and Organically Modified Interfaces in Atomic Resolution, Dalton Trans. 43 (2014) 10602-10616.

43. Q. L. Xiong, S. A. Meguid, Atomistic Investigation of the Interfacial Mechanical Characteristics of Carbon Nanotube Reinforced Epoxy Composite, Eur. Polym. J. 69 (2015) 1-15.

44. J. Zheng, R. Balasundaram, S. H. Gehrke, G. S. Heffelfinger, W. A. Goddard, S. Jiang, Cell Multipole Method for Molecular Simulations in Bulk and Confined Systems, J. Chem. Phys. 118 (2003) 5347-5355. 
45. J. E. Lennard-Jones, On the Determination of Molecular Fields, Proc. Roy. Soc. Lond. A 106 (1924) 463-477.

46. H. C. Andersen, Molecular Dynamics Simulations at Constant Pressure and/or Temperature, J. Chem. Phys. 72 (1980) 2384-2393.

47. N. A. Andrei, Scaled BFGS Preconditioned Conjugate Gradient Algorithm for Unconstrained Optimization, Appl. Math. Lett. 20 (2007) 645-650.

48. K. M. Khoda, Y. Liu, C. Storey, Generalized Polak-Ribiere Algorithm, J. Optimiz. Theory App. 75 (1992) 345-354.

49. G. Mindrila, C. Mandravel, I. Dobrica, P. Bugheanu, I. R. Stanculescu, Theoretical Study of $\beta$ and $\gamma$-Cyclodextrins Inclusion Complexes with Nineteen Atropisomeric Polychlorobiphenyls, J. Incl. Phenom. Macrocycl. Chem. 74 (2012) 137-143.

50. Y. K. Ye, S. Bai, S. Vyas, M. J. Wirth, NMR and Computational Studies of Chiral Discrimination by Amylose Tris(3,5-dimethylphenylcarbamate), J. Phys. Chem. B 111 (2007) 1189-1198.

51. R. Zhou, L. Li, B. Cheng, G. Nie, H. Zhang, Preparation and Evaluation of A Novel Bis( $\beta$-cyclodextrin)-bonded SBA-15 Chiral Stationary Phase for HPLC, Acta Chim. Sinica 72 (2014) 720-730.

52. G. Shen, X. Jia, S. Ma, T. Wang, B. Du, Z. Zhang, Resolution of Racemic -substituted Butenolide Derivatives Using A Polysaccharide Type Stationary Phase by High Performance Liquid Chromatography, J. Liq. Chromatogr. Related Technol. 37 (2014) 841-849.

53. R. Cirilli, S. Alcaro, R. Fioravanti, R. Ferretti, A. Bolasco, B. Gallinella, C. A. Faggi, Chromatographic Study on the Exceptional Enantioselectivity of Cellulose Tris(4-methylbenzoate) towards C5-chiral 4,5-Dihydro-(1H)-pyrazole Derivatives, J. Chromatogr. A 1218 (2011) 5653-5657.

54. Y. Shu, Z. S. Breitbach, M. K. Dissanayake, S. Perera, J. M. Aslan, N. Alatrash, F. M. Macdonnell, D. W. Armstrong, Enantiomeric Separations of Ruthenium (II) Polypyridyl Complexes Using HPLC with Cyclofructan Chiral Stationary Phases, Chirality 27 (2015) 64-70.

55. R. Cirilli, A. Simonelli, R. Ferretti, A. Bolasco, P. Chimenti, D. Secci, E. Maccioni, F. L. Torre, Analytical and Semipreparative High Performance Liquid Chromatography Enantioseparation of New Substituted 
1-Thiocarbamoyl-3,5-diaryl-4,5-dihydro-(1H)-pyrazoles on

Polysaccharide-based Chiral Stationary Phases in Normal-phase, Polar Organic and Reversed-phase Conditions, J. Chromatogr. A 1101 (2006) 198-203.

56. R. Ferretti, B. Gallinella, F. L. Torre, L. Zanitti, L. Turchetto, A. Mosca, R. Cirilli, Direct High-Performance Liquid Chromatography Enantioseparation of Terazosin on An Immobilised Polysaccharide-based Chiral Stationary Phase under Polar Organic and Reversed-phase Conditions, J. Chromatogr. A 1216 (2009) 5385-5390.

57. K. Zhang, N. Xue, L. Li, F. Li, Y. M. Du, Enantiomeric Separation of Azelnidipine by High Performance Liquid Chromatography with Chiral Stationary Phase, Chinese J. Chromatogr. 28 (2010) 215-217.

58. B. Gallinella, L. Bucciarelli, L. Zanitti, R. Ferretti, R. Cirilli, Direct Separation of the Enantiomers of Oxaliplatin on A Cellulose-based Chiral Stationary Phase in Hydrophilic Interaction Liquid Chromatography Mode, J. Chromatogr. A 1339 (2014) 210-213.

59. C. Zhang, L. Jin, S. Zhou, Y. Zhang, S. Feng, Q. Zhou, Chiral Separation of Neonicotinoid Insecticides by Polysaccharide-type Stationary Phases Using High-Performance Liquid Chromatography and Supercritical Fluid Chromatography, Chirality 23 (2011) 215-221.

60. R. Cirilli, R. Ferretti, B. Gallinella, E. D. Santis, L. Zanitti, F. L. Torre, High-performance Liquid Chromatography Enantioseparation of Proton Pump Inhibitors Using the Immobilized Amylose-based ChiralpakIA Chiral Stationary Phase in Normal-phase, Polar Organic and Reversed-phase Conditions, J. Chromatogr. A 1177 (2008) 105-113.

61. S. Rizzo, S. Menta, C. Faggi, M. Pierini, R. Cirilli, Influence of the Nature of Alkyl Substituents on the High-performance Liquid Chromatography Enantioseparation and Retention of New Atropisomeric 1,1'-Bibenzimidazole Derivatives on Amylase Tris(3,5-dimethylphenylcarbamate) Chiral Stationary Phase, J. Chromatogr. A 1363 (2014) 128-136.

62. P. Peluso, V. Mamane, E. Aubert, S. Cossu, High-performance Liquid Chromatography Enantioseparation of Polyhalogenated 4,4'-Bipyridines on Polysaccharide-based Chiral Stationary Phases under Multimodal Elution, J. Sep. Sci. 37 (2014) 2481-2489. 


\section{Figure Captions:}

Fig. 1. The structures of chiral compounds and CSP. The chiral center is labeled by *. Fig. 2. (a) Top view of the CSP conformation, the ball represents the R group in the structure of Chiralcel OJ in Fig. 1. (b) horizontal view of the CSP conformation and the three initial positions (labeled by green stars) of each enantiomer in the groove of CSP.

Fig. 3. The relationship between the energy and the simulation time (a) at 500K. (b) at 298K.

Fig. 4. The calculated interaction energies in seven solvents. “ $\mathbf{\Delta}$ ” represents the

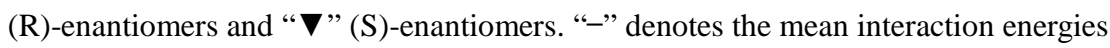
of the two enantiomers .

Fig. 5. The complex conformations of the (R)/(S)-isomers and CSP in (a) (b) 2-propanol (DC=18.62). (c) (d) Vacuum (DC=1.00). H-bond is shown by blue dotted

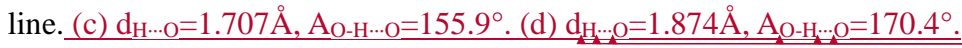

Fig. 6. The calculated interaction energies under three temperature conditions. " interaction energies of the two enantiomers.

Fig. 7. The mean energy conformation of the (R)-/(S)- complexes in (a) (b) 2-propanol under 298K(DC=18.62). (c) (d) in vacuum under 298K (DC=1.00) (e)(f) in 2-propanol under 500K (DC=18.62). (c) $\mathrm{d}_{\mathrm{H} \cdots \mathrm{O}}=1.900 \AA$, $\mathrm{A}_{\mathrm{O}-\mathrm{H} \cdots \mathrm{O}}=146.7^{\circ}$. (d) $\mathrm{d}_{\mathrm{H}} \cdots$ $\underline{\mathrm{O}}=1.828 \AA$, A $\underline{\mathrm{O}-\mathrm{H} \cdots \mathrm{O}}=168.9^{\circ}$. 